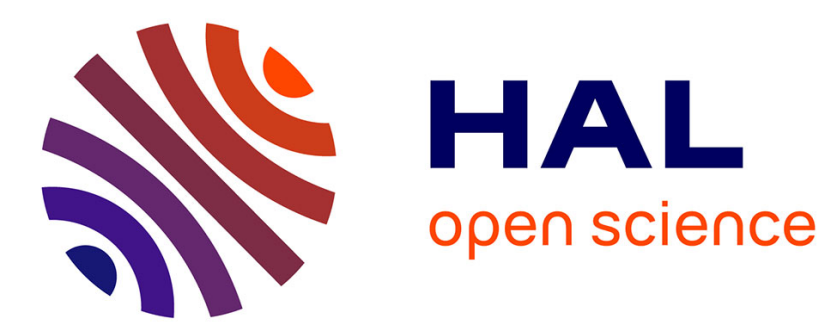

\title{
Non gaussian distributions in disordered systems studied by the cumulant expansion of EXAFS
}

\author{
G. Dalba, P. Fornasini, F. Rocca, A. Sadoc
}

\section{To cite this version:}

G. Dalba, P. Fornasini, F. Rocca, A. Sadoc. Non gaussian distributions in disordered systems studied by the cumulant expansion of EXAFS. Journal de Physique IV Proceedings, 1992, 02 (C2), pp.C2207-C2-210. 10.1051/.jp4:1992230 . jpa-00251505

\section{HAL Id: jpa-00251505 https://hal.science/jpa-00251505}

Submitted on 1 Jan 1992

HAL is a multi-disciplinary open access archive for the deposit and dissemination of scientific research documents, whether they are published or not. The documents may come from teaching and research institutions in France or abroad, or from public or private research centers.
L'archive ouverte pluridisciplinaire HAL, est destinée au dépôt et à la diffusion de documents scientifiques de niveau recherche, publiés ou non, émanant des établissements d'enseignement et de recherche français ou étrangers, des laboratoires publics ou privés. 


\title{
Non gaussian distributions in disordered systems studied by the cumulant expansion of EXAFS
}

\author{
G. DALBA, P. FORNASINI, F. ROCCA* and A. SADOC ${ }^{* *}$ \\ Dipartimento di Fisica, Universitd di Trento, Trento, Italy \\ * Centro CNR-ITC, Trento, Italy \\ ** LURE, Université Paris Sud, Orsay, France
}

\begin{abstract}
To test the effectiveness of the cumulant method for asymmetric distributions we measured and analyzed EXAFS in the anharmonic compound $\beta$-AgI from 23 to 410 $K$. The physical consistency of the so obtained cumulants has been evaluated from their temperature dependence and checked against the results from model distributions.
\end{abstract}

Résumé: Pour tester l'efficacité de la méthode des cumulants pour les distributions asymétriques, nous avons mesuré et analysé l'EXAFS de $\beta$-AgI de 23 à $410 \mathrm{~K}$. Nous avons testé la signification physique des cumulants ainsi obtenus en étudiant leur dépendence en température et en comparant avec des valeurs calculées à partier de distributions modèles.

\section{Introduction}

Non-gaussian distributions of nearest-neighbours distances are often encountered in disordered systems. Their detection and characterization can benefit from the cumulant analysis of EXAFS (extended X-ray-absorption fine-structure) /1,2/. Potentials and limits of this method have however not yet been verified on firm experimental bases: in particular the convergence properties of the cumulants series, which can seriously affect the reliability of EXAFS results, should be explored in different real physical systems.

In this paper, after reviewing the basic concepts underlying the cumulant method, we present EXAFS measurements made on $\beta$-AgI at different temperatures in the range 23 to $410 \mathrm{~K}$. $\beta$-AgI is particularly suited to check the cumulant method: the asymmetry in the distribution of $\mathrm{Ag}-\mathrm{I}$ distances is relevant already at relatively low temperatures; a previous EXAFS analysis made by Hayes et al. in the real space showed that at 300 and $371 \mathrm{~K}$ this distribution is consistent with an excluded volume model $/ 3 /$.

\section{Cumulant analysis of EXAFS}

Let $\rho(r)$ be the distribution of distances between the absorber atom and the atoms forming its s-th coordination shell. Within the single electron, single scattering, plane waves approximations, the EXAFS of the s-th coordination shell can be expressed as

$$
\chi_{s}(k)=\frac{S_{0}^{2}}{k} N_{s} \operatorname{Im}\left\{f_{s}(k, \pi) e^{2 i \delta_{1}} \int_{0}^{\infty} P(r, \lambda) e^{2 i k r} d r\right\}
$$

where $k$ is the photoelectron wavevector, $f(k, \pi)$ the backscattering amplitude, $\delta_{1}$ the central atom phaseshift, $S_{0}^{2}$ and $\lambda$ take into account intrinsic and extrinsic many-body effects, respectively. 
$P(r, \lambda)=\rho(r) \exp (-2 r / \lambda) / r^{2}$ is the effective distribution of distances. In the following we will consider only the first coordination shell, for which multiple scattering contributions are excluded.

The integral in (1) is the characteristic function of the effective distribution $P(r, \lambda)$; its logarithm can be developed in Mac Laurin series /4/, so that

$$
\int_{0}^{\infty} P(r, \lambda) e^{2 i k r} d r=\exp \left[\sum_{n=0}^{\infty} \frac{(2 i k)^{n}}{n !} C_{n}\right]
$$

The coefficients $C_{n}$ are the cumulants of the effective distribution $P(r, \lambda): C_{0}$ depends on its normalization; $C_{1}$ and $C_{2}$ are the mean value $\langle r\rangle$ and variance $\left\langle r^{2}\right\rangle-\langle r\rangle^{2}$, respectively. Higher order cumulants are zero for gaussian distributions: their values measure then the deviation of the effective distribution from a gaussian.

By substituting (2) within (1) one can see that even and odd cumulants separately determine the amplitude and phase of EXAFS signals. If one could extract from EXAFS all the significative cumulants, one would in principle be able to reconstruct the characteristic function and determine the distribution $P(r, \lambda)$ in a model-independent way.

This appealing feature of the cumulants method requires however the fulfilment of some conditions. First of all the convergence radius of the cumulant series should be longer than the useful $k$ range of the experimental EXAFS signal; the convergence should then be fast enough to require a number of cumulants not larger than the number of polynomial coefficients reasonably obtainable from an experimental EXAFS signal. Only if these conditions are fulfilled, can one utilize the cumulants obtained from EXAFS to reconstruct the low- $k$ missing part of the $\chi(k)$ signal and then, by Fourier inversion, the $P(r, \lambda)$ distribution.

The convergence properties of the cumulant series and the adequacy of EXAFS data analysis for obtaining the exact cumulants have been studied by Crozier et al. /2/ for an exponentially skewed model distribution. In this work we will face the problem of how to test experimentally the reliability of a cumulant analysis of EXAFS when dealing with an a priori unknown distribution of distances. The leading idea is to study the temperature dependence of the polynomial coefficients obtained from EXAFS analysis.

\section{Experimental results from EXAFS of $\beta$-AgI}

We measured EXAFS at the silver $\mathrm{K}$ edge in $\beta$-AgI at various temperatures in the interval 23 to $410 \mathrm{~K}$. The measurements were done at the beam line 42 of the storage ring DCI at LURE, Orsay, France. The useful $k$ range extended from 2.5 to $16.5 \AA^{-1}$.

The data analysis was done utilizing the $23 \mathrm{~K}$ spectrum as reference; we assumed that at this temperature the distribution of nearest neighbours distances is perfectly gaussian and sufficiently narrow that the cumulants of order higher than two of the effective distribution $P(r, \lambda)$ can be considered zero. The temperature-independent factor $S_{0}^{2} f_{s}(k, \pi) \exp \left(2 i \delta_{1}\right)$ was extracted from the $23 \mathrm{~K}$ reference spectrum and removed from all spectra. Following this procedure, the growth of anharmonicity with temperature is monitored by the mismatch between the maxima of imaginary part and modulus in the Fourier transforms of the EXAFS signals (Fig.1); moreover the uncertainties in EXAFS analysis arising from the resonant behaviour of the complex backscattering amplitude of iodine are strongly reduced.

The first shell contribution (Ag-I distance) was singled out at each temperature by Fourier filtering; phase and amplitude of EXAFS were then separately compared with those of the $23 \mathrm{~K}$ reference spectrum. The phase difference and the logarithm of the amplitude ratio were analyzed according to eq. (2) substituting infinite series with finite polynomials:

$$
\Phi(T)-\Phi(23)=2 k \Delta \tilde{C}_{1}-\frac{4}{3} k^{3} \Delta \tilde{C}_{3} ; \quad \ln \frac{A(T)}{A(23)}=-2 k^{2} \Delta \tilde{C}_{2}+\frac{2}{3} k^{4} \Delta \tilde{C}_{4}
$$


FIGURE 1

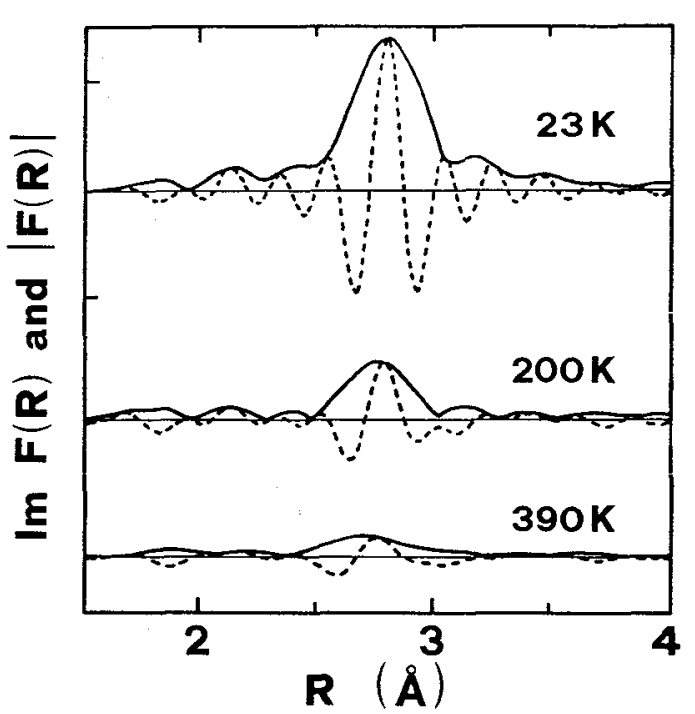

(we assumed no variation with temperature of the coordination number $N=4$ and considered negligible $\Delta C_{0}$ ). The polynomials coefficients obtained from this analysis are plotted in Fig.2 (dots) as a function of temperature. Since we considered a narrow gaussian distribution at $23 \mathrm{~K}$, $\Delta \tilde{C}_{i}=\tilde{C}_{i}(T)$ for $i=3,4$. As for $\tilde{C}_{2}$, which corresponds to the Mean Square Relative Displacement (MSRD) of absorber and backscatterer atoms, we calculated the Einstein model which best fitted the experimental slope (continuous line) and upward shifted the experimental $\Delta \tilde{C}_{2}$ values to match the theoretical ones.

The anharmonicity of $\beta$-AgI is qualitatively monitored by the growth with temperature of the $\tilde{C}_{3}$ and $\tilde{C}_{4}$ polynomial coefficients. A quantitative approach was attempted by comparing the experimental points with a parabolic behaviour, quadratic and cubic respectively, as is expected for the temperature dependence of the $C_{3}$ and $C_{4}$ cumulants (continuous lines).


FIGURE 2
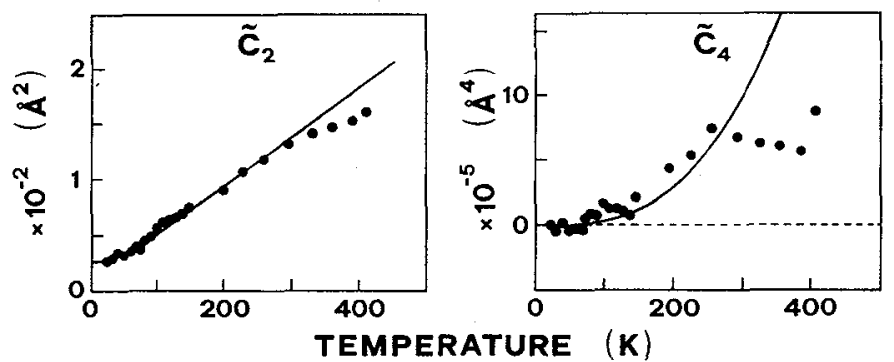

A global look at the four plots in Fig.2 allows to distinguish two regions, below and above about $300 \mathrm{~K}$. Below $300 \mathrm{~K}$ the polynomial coefficients $\tilde{C}_{i}$ behave in a physically consistent way: 
$\Delta \tilde{C}_{1}$, when the effect of the $\exp (-2 r / \lambda) / r^{2}$ factor is taken into account, is in agreement with the variation of the average interatomic distance monitored by diffraction measurements; the slope of $\tilde{C}_{2}(T)$ agrees with that obtained from an independent analysis of the $\mathrm{I} \mathrm{L}_{3}$ EXAFS /5/: its harmonic behaviour is due to the negligible average thermal expansion coefficient of $\beta-A g I ; \tilde{C}_{3}(T)$ and $\tilde{C}_{4}(T)$ are consistent with a quadratic and cubic parabolic behaviour, respectively. Above 300 $\mathrm{K}$ all polynomial coefficients deviate, more or less abruptly, from the theoretical curves.

\section{Discussion and conclusions}

The comparison between experimental points and physically consistent theoretical curves wich has been done in Fig.2 suggests that the polynomial coefficients obtained from the analysis of EXAFS in $\beta$-AgI actually correspond, below $300 \mathrm{~K}$, to the first four cumulants of the Ag-I distance distribution.

To test this conclusion, we considered the distance distribution of the excluded volume model with the parameters best fitting the $\beta$-AgI EXAFS at 300 and $371 \mathrm{~K}$, as reported by Hayes et al $/ 3 /$. The EXAFS calculated from these model distributions was then analyzed in the same way as our experimental data. The differences between the polynomial coefficients obtained from EXAFS and the exact cumulants of the original model distributions grow in passing from 300 to $371 \mathrm{~K}$ and are in agreement with the differences between experimental points and theoretical curves at the same temperatures in Fig.2.

We tried then to reconstruct the original model distributions starting from the first six cumulants. When utilizing the exact cumulants directly calculated from the distributions, a good agreement was obtained for the excluded volume distributions (better for $300 \mathrm{~K}$ than for $371 \mathrm{~K}$ ); a dramatic difference was instead found for an exponentially skewed distribution /2/ of comparable width; this seems to indicate that the convergence properties of the cumulant series are more favourable for the excluded volume than for the exponential distribution. When utilizing the first six polynomial coefficients from EXAFS analysis the agreement worsened for the excluded volume distributions, particularly at $371 \mathrm{~K}$; in particular the high $r$ tail was not completely reproduced.

These tests on model distributions showed the different behaviour, in terms of cumulants, of the excluded volume distribution, which for $\beta$ - $\mathrm{AgI}$ is upward bounded, with respect to the exponential distribution, which is unbounded. Moreover they confirm that below $300 \mathrm{~K}$ the polynomial coefficients obtained for $\beta$-AgI from EXAFS analysis are a reasonably good estimate of the true cumulants. The anomalous behaviour shown in Fig. 2 above $300 \mathrm{~K}$ is attributable to growing inadequacies of the data reduction procedure (connected with the $k$ range utilized and the reduced number of polynomial coefficients obtainable from EXAFS).

These results have been obtained for $\beta$-AgI and cannot be easily generalized. We suggest however that the adequacy of EXAFS to obtain the true cumulants can be checked, in an unknown system, by doing measurements at a sufficiently large number of temperatures and looking at the temperature dependence of the polynomial coefficients. If the true cumulants are known with sufficient accuracy, they can allow to reconstruct the temperature dependence of the distribution of distances; this could help in formulating physical models. Moreover the temperature dependence of the cumulants can give original information on the local vibrational properties of the system under study.

/1/ G. Bunker, Nucl. Instr. and Meth. 207, 437 (1983).

/2/ E.D. Crozier, J.J. Rehr, and R. Ingalls, in $X$-ray absorption, ed. by D.C. Koningsberger and R. Prins, John Wiley \& Sons, New York (1988).

/3/ T.M. Hayes, J.B. Boyce and J.L. Beeby, J. Phys. C: Solid State Phys. 11, 2931 (1978).

/4/ H. Cramér, Mathematical methods of statistics, Princeton Univ. Press (1966).

/5/ G. Dalba, P. Fornasini, F. Rocca, and S. Mobilio, Phys. Rev. B 41, 9668 (1990); G. Dalba, P. Fornasini, and F. Rocca, J. Phys. C, in print (1992). 